Airway assessment by a SALT team was performed in 4 patients within $48 \mathrm{~h}$ of admission. None of the patients underwent video fluoroscopy. An initial dietetic review within $72 \mathrm{~h}$ of admission was observed in 6 patients (18\%). Median hospital stay was 8 days and in hospital mortality was $41 \%$.

Conclusion The study demonstrates a significant inconsistency in the initial management of patients hospitalised with AP. The lack of early intervention by SALT and dietetic services and routine video fluoroscopy use in the majority of patients is of concern. A specific guideline with an evidence based diagnostic pathwayand managementis needed for patients at high risk for AP.

\section{P87 KLEBSIELLA PNEUMONIAE SURVIVAL ON PLASTIC VALVED HOLDING CHAMBER BODIES}

MJ Sanders, R Bruin. Clement Clarke International Ltd., Harlow, UK

\subsection{6/thoraxjnl-2014-206260.228}

Introduction and objectives Klebsiella pneumoniae (KPN) is an opportunistic pathogen for patients with chronic pulmonary disease. In-use single time-point sampling of valved holding chamber spacer (VHC) bodies has shown evidence (Cohen and Cohen, JAMA 2003, 290:195-196) of bacterial contamination particularly with Pseudomonas species and Klebsiella. We are not aware of multi time-point bacterial survival research and, accordingly, have assessed in vitro two plastic VHC body materials which are in common use in the UK.

Methods Test pieces, in $\mathrm{n}=5$ sub-group samples (Samples), of polystyrene (sterile Control) and of VHC bodies of AeroChamber Plus, Trudell Medical International (ACP) and A2A Spacer, Clement Clarke International Ltd. (A2A) were equilibrated for $24 \mathrm{~h}$ at $20^{\circ} \mathrm{C} / 65 \%$ relative humidity $(\mathrm{RH})$. Samples were inoculated with $100 \mathrm{muL}$ distilled-water aliquots of KPN $3.6 \times 10^{6}$ cells. $\mathrm{mL}^{-1}$ and incubated at $20^{\circ} \mathrm{C}$ in $65 \% \mathrm{RH}$ chambers. KPN surface survival was measured at $0,24,48$ and $72 \mathrm{~h}$ time-points using a method based on ISO Standard 22196:2007 (Askew, Efficacy Assessment of Treated Articles: A Guidance, February 2014 http://dx.doi.org/10.6027/NA2014-904) and total viable count enumerated. $72 \mathrm{~h}$ data $\left(\log _{10}\right.$ Colony Forming Units $(\mathrm{CFU}) . \mathrm{cm}^{-2}$ ) were analysed using one-way analysis of variance.

Results Geometric mean CFU. $\mathrm{cm}^{-2}$ data are given in the table. Control and ACP KPN populations declined to the $48 \mathrm{~h}$ time-point, thereafter increasing (Control) and remaining constant (ACP). A2A KPN population showed a constant decline, with no re-growth. At $72 \mathrm{~h}, \log _{10}$ CFU KPN data for A2A were significantly smaller ( $\mathrm{p}<$ 0.05 ) compared to ACP (-0.47 difference, $95 \%$ CI -0.81 to -0.13 ) and to Control (0.74 difference, $95 \%$ CI 0.41 to 1.08 ).

Conclusions We interpret the $72 \mathrm{~h}$ increase in Control KPN as the outcome of the initial decline creating dead cell matter that acted as a nutrient source. The stability (ACP) and significant decline (A2A) in KPN is therefore an interesting finding. We subscribe the latter to the presence of an antimicrobial additive in

\section{Abstract P87 Table 1}

\begin{tabular}{lllll}
\hline \multirow{5}{*}{$\begin{array}{llll}\text { Sample } \\
(\mathrm{n}=5)\end{array}$} & \multicolumn{4}{l}{ Geometric mean KPN CFU.cm } \\
\cline { 2 - 5 } & \multicolumn{2}{l}{ Contact time (hours) } & & \\
\hline Control & $1.8 \times 10^{5}$ & $4.7 \times 10^{5}$ & $2.8 \times 10^{4}$ & $4.8 \times 10^{4}$ \\
ACP & $1.8 \times 10^{5}$ & $5.4 \times 10^{4}$ & $2.7 \times 10^{4}$ & $2.5 \times 10^{4}$ \\
A2A & $1.8 \times 10^{5}$ & $5.1 \times 10^{4}$ & $1.7 \times 10^{4}$ & $8.6 \times 10^{3}$ \\
\hline
\end{tabular}

the body polymer material. The clinical implications of these findings are relevant to VHC hygiene and patient health, and require further investigation.

\section{Smoothing the process: clinical management of COPD and bronchiectasis}

\section{P88 AN EVALUATION OF THE PATIENTS PATHWAY TO ACCIDENT AND EMERGENCY (A AND E) OR INPATIENT ADMISSION FOLLOWING AN EXACERBATION OF ASTHMA OR CHRONIC OBSTRUCTIVE PULMONARY DISEASE (COPD) IN A LARGE TEACHING HOSPITAL}

M Naqvi, H Khachi. Barts Health NHS Trust, London, UK

10.1136/thoraxjnl-2014-206260.229

Introduction Asthma and COPD account for a significant proportion of inpatient admissions in the UK. A national review of asthma deaths found that a significant proportion of patients die without seeking medical assistance or before emergency medical care could be provided. ${ }^{1}$

Objectives Establish the pathway that patients undertake to access care in the lead up to an A and E attendance and/or inpatient admission.

Method Patients attending $\mathrm{A}$ and $\mathrm{E}$ and/or following an inpatient admission due to an exacerbation of asthma or COPD were reviewed by a respiratory pharmacist during weekday working hours. Patients were identified during post take ward rounds and using hospital electronic systems. All analyses were conducted using SPSS 22. Ethics approval was not required.

Results Over the six-month period, 539 (138 asthma and 403 COPD) presentations for exacerbations of asthma and COPD were reviewed. As Table 1 shows, only 48\% $(\mathrm{n}=66)$ and $46 \%$ $(\mathrm{n}=185)$ of asthma and COPD patients respectively, received medical attention and/or had an active intervention (e.g. administration of rescue pack of oral corticosteroids and/or antibiotics) prior to presenting to hospital. The remaining 52\% (n $=72)$ and 54\% ( $\mathrm{n}=218)$ respectively either did not seek medical attention or were unable to be reviewed (e.g. unable to obtain an appointment with their general practitioner, GP) prior to their attendance. The results also show that the majority of patients were registered with a GP.

Conclusion Despite the majority of patients having access to a GP, a significant proportion of asthma and COPD patients either did not seek medical attention prior to presenting to hospital, or were unable to be reviewed by their GP. These findings correlate with those found in the national review of asthma deaths. ${ }^{1}$ At a time of increasing demands on healthcare resources, these results pose the question of how we can better triage patients to

\begin{tabular}{lll}
$\begin{array}{l}\text { Abstract P88 Table } 1 \\
\text { Admission }\end{array}$ & Pathways to $\mathrm{A}$ and $\mathrm{E}$ and/or Inpatient \\
\hline & Asthma (n=138) & COPD $(\mathbf{n}=403)$ \\
\hline $\begin{array}{l}\text { Registered with GP } \\
\begin{array}{l}\text { Patients who sought medical } \\
\text { attention and/or active intervention }\end{array}\end{array}$ & $135(98 \%)$ & $392(97 \%)$ \\
$\begin{array}{l}\text { Patients who sought medical } \\
\text { attention but did not receive it }\end{array}$ & $66(48 \%)$ & $185(46 \%)$ \\
$\begin{array}{l}\text { Patients who did not seek } \\
\text { medical attention and/or active intervention }\end{array}$ & $52(38 \%)$ & $176(14 \%)$ \\
\hline
\end{tabular}


appropriate care settings to minimise unscheduled care and improve patient access and care.

\section{REFERENCE}

1 Royal College of Physicians. Why asthma still kills: the National Review of Asthma Deaths (NRAD). Confidential Enquiry Report. May 2014

\section{P89 ATTENDANCE OF SECONDARY CARE RESPIRATORY OUTPATIENT APPOINTMENTS IN ILLICIT DRUG USERS WITH RECURRENT HOSPITAL ADMISSIONS WITH 'COPD' AT A CITY CENTRE TEACHING HOSPITAL}

R Huang, AM Collins, N Williams, N Garner, T Perry, H Burhan. Royal Respiratory Research (RRR), The Royal Liverpool and Broadgreen University Hospital Trust, Liverpool, UK

\subsection{6/thoraxjnl-2014-206260.230}

Introduction Non-attendance at outpatient appointments (OPA) costs the NHS an estimated $£ 600 \mathrm{~m}$ a year, with over 94,000 missed (first) OPAs in England from 2013 to 2014. ${ }^{1}$ We reviewed the arrangement and attendance of OPAs for illicit drug smokers, after hospital re-admission with an 'exacerbation of chronic obstructive pulmonary disease (COPD)'.

Methods All illicit drug smokers re-admitted between January 2009 and September 2011 with a presumptive diagnosis of 'exacerbation of COPD' were included. Planned respiratory OPAs were reviewed retrospectively from our COPD admission database to determine the number attended or unattended. Unattended OPAs were classified as (a) hospital cancellation (b) patient cancellation (c) patient did not attend (DNA) or (d) 'unknown'.

Results Of 89 patients, no OPA was arranged in 28 (31.5\%). 334 respiratory appointments were made for 61 patients (mean $=5.5$ per patient); of these, only $86(25.7 \%)$ were attended (see Table).

Conclusion High recurrent admission rates suggests that these patients should all have specialist respiratory OPAs arranged at discharge, with the aim of preventing re-admission and improving their respiratory health. In our cohort we noted poor OPA attendance with a DNA rate of $52.0 \%$ compared with around $8.6 \%$ for first OPAs overall in England in 2012. ${ }^{2}$ This suggests alternative approaches are needed in order to engage with these patients such as community based secondary care outreach services. We will now study the effects of an intensive community-based secondary care outreach services; involving smoking cessation, targeted pulmonary rehabilitation, specialist respiratory (consultant and nurse) involvement, vaccination, inhaler technique reviews, medication concordance checks/ prescription and health trainers.

\section{REFERENCES}

1 Department of Health (2014). Referrals and Attendances for Outpatient Appointments: Hospital Activity Statistics. London: DH

2 Department of Health (2013). NHS inpatient elective admission events and outpatient referrals and attendances, quarter ending December 2012. Available from: http://www.england.nhs.uk/statistics/2013/02/22/nhs-inpatient-elective-admissionevents-and-outpatient-referrals-and-attendances-quarter-ending-december-2012

\begin{tabular}{lll} 
Abstract P89 Table 1 & & \\
\hline Not attended $(n=248)$ & & \\
Reason & Hospital cancelled & $92(37.1 \%)$ \\
& Patient cancelled & $8(3.2 \%)$ \\
& DNA & $129(52.0 \%)$ \\
& 'Unknown' & $19(7.7 \%)$ \\
\hline
\end{tabular}

\section{P90 CATCH - COMMUNITY ACCESS TO CT CHEST}

${ }^{1} \mathrm{CS}$ Moorcroft, ${ }^{1} \mathrm{G}$ Kamalatharen, ${ }^{2} \mathrm{~S}$ Elliot, ${ }^{3} \mathrm{~A}$ Walsham, ${ }^{3} \mathrm{~A}$ Sharman, ${ }^{3} \mathrm{SCO}$ Taggart. ${ }^{1}$ Manchester Medical School, Manchester, UK; ${ }^{2}$ Salford Clinical Commissioning Group, Salford, UK; ${ }^{3}$ Salford Royal NHS Foundation Trust, Salford, UK

\subsection{6/thoraxjnl-2014-206260.231}

Introduction Rates of lung cancer diagnosis for two week wait (2WW) referrals are low although referrals are increasing. Many 2 WW's are potentially exposed to unnecessary anxiety as the referral requires the G. P. to inform the patient of the possibility of cancer. CATCH (Community Access To CT Chest) is a new protocol of care that has been developed by the Salford lung cancer team in collaboration with the Salford C. C. G. whereby abnormal "low risk" CXR reports are communicated to G. P.s with instructions for them to request a CT scan, which is then fast tracked allowing rapid performance and reporting of the scan with appropriate advice to the GP.

Methods A d-base was set up to capture the performance of CATCH from its introduction on 05.02.2014 to 05.07.2014. Demographic details were collected for dates of CXR, CXR report, CT request, CT report, relevant outcomes and 2WW activity for same time (2011-2014). Participating patients were interviewed by telephone using a structured questionnaire (supported by a postal questionnaire for non-responders).

Results 53 patients underwent an abnormal CXR with advice to enter into the CATCH protocol and of these 7 bypassed $\mathrm{CATCH}$ having been referred directly into the $2 \mathrm{WW}$ system by their G. P.s. For the 46 patients completing CATCH, seven $(15 \%)$ urgent $2 \mathrm{WW}$ referrals were recommended. In the remaining 39 patients, 28 required no follow up, 9 non-urgent referral to the chest clinic and 2 repeat community CXRs. Timelines for performance of CT scans were acceptable (see Table) and detected cancer in 5/46 (10.9\%) and were normal in $8 / 46$ (17.4\%). 23-26 patients interviewed to date rated the service overall as either very good or excellent. During same points in 2011, 2012, 2013 and 2014

2WW numbers were $69,84,89$ and 81 respectively. Conclusions Our provisional data support the role of $\mathrm{CATCH}$ as a new system of care for managing "low risk "CXR reports that might otherwise be referred into growing $2 \mathrm{WW}$ clinics. Thus far, the protocol moves at a rapid pace and has been well received by the patient (although we await the results of the postal survey in due course).

\section{Abstract P90 Table 1 Mean time for various CATCH outcomes

CXR performed to CXR report 3.1 days

CXR report to CT request 5.1 days \\ $\mathrm{CT}$ request to $\mathrm{CT}$ appointment 5.8 days \\ CXR report to $\mathrm{CT}$ appointment 13.0 days \\ P91 DEVELOPMENT AND IMPLEMENTATION OF A STRUCTURED, ANNUAL 'COMPREHENSIVE RESPIRATORY ASSESSMENT' FOR INDIVIDUALS WITH ADVANCED COPD}

N Toms, NJ Greening, R Free, JE Williams, RA Evans, MC Steiner. Glenfield Hospital, Leicester, UK

10.1136/thoraxjnl-2014-206260.232

Introduction Disease burden, polypharmacy, co-morbidities and complex social needs are significant in patients with advanced COPD and predict morbidity, mortality and health care 